\title{
Do we need FDG-PET/CT to assess atherosclerosis?
}

\author{
Nicolò Pipitone $^{1} \cdot$ Carlo Salvarani $^{1} \cdot$ Annibale Versari $^{2}$
}

Published online: 18 November 2016

(C) Springer-Verlag Berlin Heidelberg 2016

The evaluation of atherosclerosis progression is a key issue in cardiovascular prevention because it could help in reducing cardiovascular morbidity and mortality.

In this issue of the European Journal of Nuclear Medicine and Molecular Imaging, Fayad et al. report on the results of a prospective substudy of the Dal-Plaque trial [1], which investigated the efficacy and safety of the cholesteryl ester transfer protein modulator dalcetrapib. The authors provide evidence that short-term (6 months) changes in arterial inflammation as assessed by ${ }^{18} \mathrm{~F}$-fluorodeoxyglucose positron emission tomography (FDG-PET) were associated with longer-term (24 months) structural changes of atherosclerosis evaluated by MRI. The findings of this study confirm the capacity of PET to predict structural changes of atherosclerosis demonstrated by Dal-Plaque, and improve on the results of the original study, in which total vessel areas were assessed by MRI, while in this substudy mean wall area and thickness were determined. Total vessel area is not fully appropriate to assess atherosclerosis progression because an increase in the vessel lumen could theoretically occur without concomitant changes in wall area or thickness, which are more stringent outcome measures.

This Editorial Commentary refers to the article http://dx.doi.org/10.1007/ s00259-016-3524-0.

Annibale Versari

versari.annibale@asmn.re.it

1 Department of Rheumatology, Arcispedale S. Maria Nuova-IRCCS of Reggio Emilia, Reggio Emilia, Italy

2 Department of Nuclear Medicine, Arcispedale S. Maria Nuova-IRCCS of Reggio Emilia, Reggio Emilia, Italy
PET has been shown to be sensitive and reproducible in delineating vascular inflammation in various disorders. In vasculitis, a linear, smooth vascular FGD uptake extending over long vessel segments is typically observed [2]. In contrast, atherosclerosis is characterized by an irregular, more limited, "spotty" pattern [2], which corresponds to vascular areas more heavily infiltrated by macrophages, i.e., to unstable atheroma, which is potentially thrombogenic [3]. Consistent with this concept, PET has been demonstrated to be able to predict adverse cardiovascular events [4].

The results of this substudy further strengthen the relevance of arterial inflammation as assessed by PET to the subsequent development of atherosclerotic changes, but also raise some questions. First, why did baseline vascular FDG uptake not predict structural changes at 24 months? The authors claim that the pharmacological intervention may have acted as confounder, but this explanation conflicts with the observation that dalcetrapib did not reduce carotid artery inflammation relative to placebo. Moreover, inflammatory changes at 3 months also failed to predict later structural changes, suggesting that the delta between baseline and subsequent inflammation may have greater predictive power for vascular changes. This would be in keeping with the concept, well-established in oncology, that changes in tumor SUV following treatment may have better prognostic value than baseline SUV [5]. The specific pharmacological agent investigated may also be relevant to the optimal time frame required to detect significant inflammatory changes, which may have implications for future clinical trials. Another important observation [1] is that inflammation in the carotid arteries appears to be more sensitive than the aorta in predicting structural changes; this finding, too, may have relevance to future studies on pharmacological agents for atherosclerosis. 
No association was found in this substudy between changes in biomarkers of inflammation and structural vascular changes, despite the fact that the $\mathrm{C}$-reactive protein is an established marker for atherosclerosis progression. This finding suggests that PET has a complementary role to the measurement of inflammatory biomarkers, and specifically that local inflammation may be more important that systemic inflammation to determine future arterial wall abnormalities.

In conclusion, this study underscores the role of PET as a promising outcome measure in future trials on agents that target vascular inflammation, although the optimal time points at which PET should be performed remain sub judice and may differ for different agents. As the authors state, assessing vascular inflammation by PET may facilitate the evaluation of the efficacy of novel candidate molecules in future clinical trials and allow investigators to move more confidently to phase III clinical trials.

\section{References}

1. Fayad ZA, Mani V, Woodward M, et al. Safety and efficacy of dalcetrapib on atherosclerotic disease using novel non-invasive multimodality imaging (dal-PLAQUE): a randomised clinical trial. Lancet. 2011;378(9802):1547-59.

2. Pipitone N, Versari A, Salvarani C. Role of imaging studies in the diagnosis and follow-up of large-vessel vasculitis: an update. Rheumatology (Oxford). 2008;47(4):403-8.

3. Buscombe JR. Exploring the nature of atheroma and cardiovascular inflammation in vivo using positron emission tomography (PET). Br J Radiol. 2015;88(1053):20140648.

4. Figueroa AL, Abdelbaky A, Truong QA, et al. Measurement of arterial activity on routine FDG PET/CT images improves prediction of risk of future CV events. JACC Cardiovasc Imaging. 2013;6(12): 1250-9.

5. Casasnovas RO, Meignan M, Berriolo-Riedinger A, et al. SUVmax reduction improves early prognosis value of interim positron emission tomography scans in diffuse large B-cell lymphoma. Blood. 2011;118(1):37-43. 